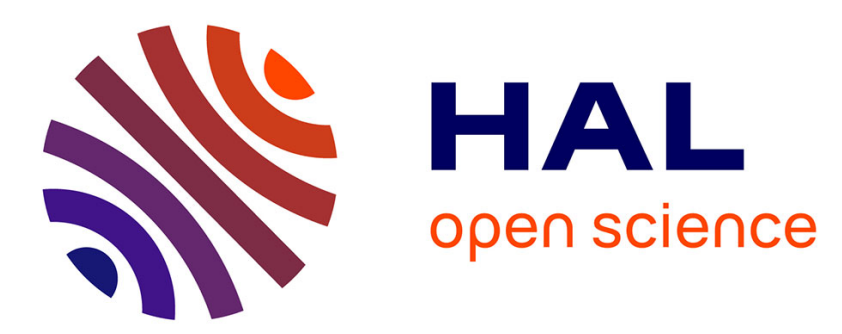

\title{
Active Decentralized Scale Estimation for Bearing-Based Localization
}

\author{
Riccardo Spica, Paolo Robuffo Giordano
}

\section{To cite this version:}

Riccardo Spica, Paolo Robuffo Giordano. Active Decentralized Scale Estimation for Bearing-Based Localization. IEEE/RSJ Int. Conf. on Intelligent Robots and Systems, IROS'16, Oct 2016, Daejeon, South Korea. hal-01355789

\section{HAL Id: hal-01355789 \\ https://hal.inria.fr/hal-01355789}

Submitted on 24 Aug 2016

HAL is a multi-disciplinary open access archive for the deposit and dissemination of scientific research documents, whether they are published or not. The documents may come from teaching and research institutions in France or abroad, or from public or private research centers.
L'archive ouverte pluridisciplinaire HAL, est destinée au dépôt et à la diffusion de documents scientifiques de niveau recherche, publiés ou non, émanant des établissements d'enseignement et de recherche français ou étrangers, des laboratoires publics ou privés. 


\title{
Active Decentralized Scale Estimation for Bearing-Based Localization
}

\author{
Riccardo Spica and Paolo Robuffo Giordano
}

\begin{abstract}
In this paper, we propose a novel decentralized active perception strategy that maximizes the convergence rate in estimating the (unmeasurable) formation scale in the context of bearing-based formation localization for robots evolving in $\mathbb{R}^{3} \times \mathcal{S}^{1}$. The proposed algorithm does not assume presence of a global reference frame and only requires bearing-rigidity of the formation (for the localization problem to admit a unique solution), and presence of (at least) one pair of robots in mutual visibility. Two different scenarios are considered in which the active scale estimation problem is treated either as a primary task or as a secondary objective with respect to the constraint of attaining a desired bearing formation. The theoretical results are validated by realistic simulations.
\end{abstract}

\section{INTRODUCTION}

Multi-robot systems have proven to be very effective in many applications such as exploration and mapping [1], search and rescue [2], and load transportation [3]. Such systems can, indeed, significantly increase the robustness and reliability in realizing sophisticated tasks by distributing the workload and thus reducing the complexity of each agent in the team. To this end, a common requirement is to employ decentralized estimation/control algorithms so as to avoid the risks associated with the possible failure of a central node and the higher computational load required to process all the available information in a centralized fashion.

To enable a collaborative sensing/decision making, it is crucial for the agents to share information. A sufficient level of connectivity of the communication graph must therefore be guaranteed. In addition to this, when the available measurements are expressed in the robot local body frames, it is also essential for the group to agree over a common shared frame for exchanging the individually acquired information. When access to a common global reference frame is possible, as when employing GPS or MoCap systems, the task is significantly simplified [3]-[7]. In unstructured GPS-denied environments, instead, the robots can only rely on measurements expressed in a local reference frame and the problem becomes considerably more challenging [8][10]. For instance, in the case of bearing measurements from onboard cameras, the authors of [11] overcome this problem by assuming all measurements to be reciprocal. This assumption, however, can be hard to be met in practice because of field of view limitations.

In this context, the theory of formation rigidity [12] has proven to be an effective theoretical framework for the analysis and resolution of the cooperative localization problem

R. Spica and P. Robuffo Giordano are with the CNRS at Irisa and Inria Rennes Bretagne Atlantique, Campus de Beaulieu, 35042 Rennes Cedex, France riccardo.spica, prgeirisa.fr

This research was supported by the ANR, Project ANR-14-CE27-0007 SenseFly. and of its dual, i.e., the formation control problem, when relying on local sensing such as relative distance [13]-[15] or bearing [7], [8], [16], [17] measurements. This latter case is particularly relevant for many applications since relative bearings can be directly acquired using onboard cameras, which is a ubiquitous sensor modality especially in mobile robotics.

As well known, when only relying on bearing measurements, the metric scale of the formation cannot be recovered/controlled since bearings are scale invariant. Knowledge of the actual formation scale is, nevertheless, necessary for ensuring convergence of bearing-based localization schemes in case of moving agents [18] and, more in general, for a safe navigation in presence of obstacles. To recover the scale, an additional "metric" measurement must then be employed.

One convenient possibility is to assume presence of (at least) one distance measurement among a pair of agents [8], [10], [18], [19]. As shown in [10] this is, indeed, sufficient to recover the scale of the whole formation in a decentralized way. Measuring inter-agent distances does, however, require additional sensors, thus increasing the system complexity.

An alternative solution is to attempt to recover the (unmeasurable) inter-agent distances by exploiting structure from motion (SfM) algorithms (see, e.g. [20]) that can fuse online the available bearing measurements and the (metric) bodyframe linear velocity of the robots. Indeed, in any typical scenario this latter quantity can be considered as 'known' as well, since it is eventually required for implementing any feedback control of the robot own motion through space. In this paper we will then consider this second possibility.

A number of works has addressed the problem of retrieving the robot inter-distances, or the formation scale, by fusing the measured bearings and the agent motion. For instance in [19] the authors propose a closed-form solution to fuse bearing measurements taken from different vantage points with known relative displacements. The processing, however, is done in a centralized way. A decentralized solution for fusing velocity and bearing measurements was, instead, proposed in [21].

None of these works, however, considers the problem of actively ensuring that the agent trajectories are "sufficiently informative" for the scale estimation to converge. Indeed, as well known, the accuracy of any SfM algorithm is strongly affected by the trajectory followed by the robot/camera during the estimation process [22], [23]. Guaranteeing a sufficient level of excitation/observability during motion is, therefore, of paramount importance. An interesting attempt to actively improve cooperative estimation performance was proposed in [24], but by considering relative position sensors 
and using a centralized control law.

Compared to this state-of-the-art, the goal of this paper is to allow a team of quadrotor UAVs equipped with onboard cameras to collectively localize (with the correct scale factor and in a decentralized way) in a common shared frame by fusing the measured inter-robot bearings and the robot own velocities, with both quantities assumed to be only expressed in the local body frames of the robots. To this end, we extend the active SfM scheme detailed in [25] (for a single camera observing a static scene) to the case at hand for estimating a subset of (at least one) inter-agent distances. Coupled with the bearing-based localization algorithm proposed in [18], this allows to retrieve a correctly scaled estimation of the formation configuration.

One novelty of our strategy is that the convergence rate of the estimated distances is optimized online by acting on the robot motion. Moreover, both the estimation and control algorithms are fully decentralized and consider a directed measurement graph with the only additional requirement of containing at least one reciprocal link.

Two different scenarios are considered. In the first, the cooperative localization is the only task assigned to the robots which are, then, free to move in the most suitable way for optimizing the scale estimation performance. In the second (more realistic) scenario, a primary task is assigned in terms of reaching (and maintaining) a desired bearing formation, and the SfM performance must be optimized in the null space of the desired bearing formation constraint.

The paper is organized as follows. In Sect. II we introduce the agent motion and sensing models. In Sect. III we summarize previous results in bearing-based decentralized localization [18] and active SfM [25]. In Sect. IV we describe our core contribution: a decentralized active perception algorithm that maximizes the convergence rate of relative distance estimations, possibly while also considering a formation control task. Finally, in Sect. V, we validate our approach with realistic simulations and, in Sect. VI, we draw some conclusions and discuss possible future work.

\section{PRELIMINARIES}

\section{A. Motion and sensing model}

Let us consider a formation of $N$ quadrotor UAVs. Following [8], [18], [26], the dynamics of the $i$-th robot can be modeled, for the purpose of this work, as ${ }^{1}$

$$
\left[\begin{array}{c}
\dot{\boldsymbol{p}}_{i} \\
\dot{\psi}_{i}
\end{array}\right]=\left[\begin{array}{cc}
\boldsymbol{R}_{i} & 0 \\
\mathbf{0}_{3} & 1
\end{array}\right]\left[\begin{array}{c}
\boldsymbol{u}_{i} \\
w_{i}
\end{array}\right]
$$

where $\boldsymbol{p}_{i} \in \mathbb{R}^{3}$ is the robot position in the world frame, $\psi_{i} \in \mathcal{S}^{1}$ is the yaw angle and $\boldsymbol{R}_{i}=\boldsymbol{R}_{z}\left(\psi_{i}\right)$ is the canonical rotation around the $\boldsymbol{z}$-axis. The quantities $\boldsymbol{u}_{i} \in \mathbb{R}^{3}$ and $w_{i} \in \mathbb{R}$ are the body-frame linear velocity and yaw rate which are assumed to be known and controllable. The robot configuration is given by $\boldsymbol{q}_{i}=\left(\boldsymbol{p}_{i}, \psi_{i}\right) \in \mathbb{R}^{3} \times \mathcal{S}^{1}$. The configuration of the whole formation is represented by

\footnotetext{
${ }^{1}$ The simulations in Sect. V will demonstrate the robustness of our proposed solution w.r.t. this simplification.
}

$\boldsymbol{q}=(\boldsymbol{p}, \boldsymbol{\psi}) \in\left(\mathbb{R}^{3} \times \mathcal{S}^{1}\right)^{N}$, with $\boldsymbol{p}=\left(\boldsymbol{p}_{1}, \ldots, \boldsymbol{p}_{N}\right) \in \mathbb{R}^{3 N}$ and $\boldsymbol{\psi}=\left(\psi_{1}, \ldots, \psi_{N}\right) \in \mathcal{S}^{N}$.

We assume presence of an onboard calibrated camera that allows a robot $i$ to measure its relative bearing w.r.t. a robot $j$ expressed in its local body-frame, i.e.,

$$
\boldsymbol{\beta}_{i j}=\boldsymbol{R}_{i}^{T} \frac{\boldsymbol{p}_{j}-\boldsymbol{p}_{i}}{\left\|\boldsymbol{p}_{j}-\boldsymbol{p}_{i}\right\|}=\boldsymbol{R}_{i}^{T} \frac{\boldsymbol{p}_{i j}}{d_{i j}} \in \mathbb{S}^{2}
$$

where $\mathbb{S}^{2}$ is the surface of the unit sphere in $\mathbb{R}^{3}, \boldsymbol{p}_{i j}=\boldsymbol{p}_{j}-\boldsymbol{p}_{i}$ and $d_{i j}=\left\|\boldsymbol{p}_{i j}\right\|$.

\section{BEARING-BASED LOCALIZATION}

\section{A. Summary of Bearing Rigidity Framework in $\mathbb{R}^{3} \times \mathcal{S}^{1}$}

In this section we summarize the bearing rigidity framework proposed in [10] and extended in [18] to the case of non-stationary agents in $\mathbb{R}^{3} \times \mathcal{S}^{1}$.

Let us consider a directed graph $\mathcal{G}=(\mathcal{V}, \mathcal{E})$ with vertex set $\mathcal{V}=1 \ldots N$ and edge set $\mathcal{E} \subseteq \mathcal{V} \times \mathcal{V}$ [27]. An edge $(i, j)$ represents the possibility for agent $i$ to measure its relative bearing $\boldsymbol{\beta}_{i j}$ w.r.t. agent $j$ as in (2). Note that presence of edge $(i, j)$ does not imply that of edge $(j, i)$, i.e., the bearing measurements are not required to be reciprocal. Let us, then, also define an undirected subgraph $\mathcal{G}_{u}=\left(\mathcal{V}_{u}, \mathcal{E}_{u}\right)$ that contains all reciprocal measurements in $\mathcal{G}$ and the associated vertices, i.e. $\mathcal{E}_{u}=\{e \in \mathcal{E}:(i, j) \in \mathcal{E},(j, i) \in \mathcal{E}, i<j\} \subseteq \mathcal{E}$ and $\mathcal{V}_{u}=\left\{i \in \mathcal{V}:[(i, j)] \in \mathcal{E}_{u}\right\} \subseteq \mathcal{V}$ where $[\cdot]$ represents the equivalence class. Finally we assume, that $i$ and $j$ can communicate if either $(i, j) \in \mathcal{E}$ or $(j, i) \in \mathcal{E}$. We also assume, as in most of the related works, that the bearing-to-robot correspondence map is known thanks to, e.g., distinctive markers attached to the robots or the use of a registration algorithm such as those proposed in [28].

A framework (i.e. a formation) is defined as the pair $(\mathcal{G}, \boldsymbol{q})$. The (directed) bearing function associated to a framework $(\mathcal{G}, \boldsymbol{q})$ is the map

$$
\boldsymbol{\beta}_{\mathcal{G}}:\left(\mathbb{R}^{3} \times \mathcal{S}^{1}\right)^{N} \mapsto\left(\mathbb{S}^{2}\right)^{|\mathcal{E}|}, \boldsymbol{\beta}_{\mathcal{G}}(\boldsymbol{q})=\left(\boldsymbol{\beta} e_{1}, \ldots, \boldsymbol{\beta} e_{|\mathcal{E}|}\right),
$$

where $e_{i} \in \mathcal{E}$ represents a directed edge in $\mathcal{G}$ according to any chosen labeling. The (directed) world-frame bearing rigidity matrix is the Jacobian $\boldsymbol{B}_{\mathcal{G}}^{\mathcal{W}}(\boldsymbol{q})=\frac{\partial \boldsymbol{\beta}_{\mathcal{G}}(\boldsymbol{q})}{\partial \boldsymbol{q}} \in \mathbb{R}^{3|\mathcal{E}| \times 4 N}$ that allows to express the measurement dynamics in terms of the agents world-frame velocities $\dot{\boldsymbol{q}}=(\dot{\boldsymbol{p}}, \dot{\psi})$, i.e. $\dot{\boldsymbol{\beta}}_{\mathcal{G}}=$ $\mathcal{B}_{\mathcal{G}}^{\mathcal{W}}(\boldsymbol{q}) \dot{\boldsymbol{q}}$. Similarly, the body-frame bearing rigidity matrix

$$
\dot{\boldsymbol{\beta}}_{\mathcal{G}}=\mathcal{B}_{\mathcal{G}}^{\mathcal{W}}(\boldsymbol{q})\left[\begin{array}{cc}
\operatorname{diag}\left(\boldsymbol{R}_{i}\right) & \mathbf{0} \\
\mathbf{0} & \boldsymbol{I}_{N}
\end{array}\right]\left[\begin{array}{c}
\boldsymbol{u} \\
\boldsymbol{w}
\end{array}\right]=\boldsymbol{B}_{\mathcal{G}}(\boldsymbol{q})\left[\begin{array}{c}
\boldsymbol{u} \\
\boldsymbol{w}
\end{array}\right]
$$

relates the measurement dynamics to the agent body-frame velocities. The $k$-th row block of $\boldsymbol{B}_{\mathcal{G}}(\boldsymbol{q})$, associated to the edge $e_{k}=(i, j)$, has the following expression

$$
\left[\begin{array}{lllccccc}
\mathbf{0} & -\frac{1}{d_{i j}} \boldsymbol{P}_{i j} & \mathbf{0} & \frac{1}{d_{i j}} \boldsymbol{P}_{i j}{ }^{i} \boldsymbol{R}_{j} & \mathbf{0} & -\boldsymbol{S} \boldsymbol{\beta}_{i j} & \mathbf{0}
\end{array}\right],
$$

where $\boldsymbol{P}_{i j}=\boldsymbol{I}_{3}-\boldsymbol{\beta}_{i j} \boldsymbol{\beta}_{i j}^{T}, \boldsymbol{S}$ is the skew-symmetric matrix built with the components of $\boldsymbol{e}_{3}=(0,0,1)$ and ${ }^{i} \boldsymbol{R}_{j}=$ $\boldsymbol{R}_{z}\left(\psi_{i j}\right)$ with $\psi_{i j}=\psi_{j}-\psi_{i}$.

A framework $(\mathcal{G}, \boldsymbol{q})$ is termed infinitesimally bearing rigid (IBR) at $\boldsymbol{q}$ if, letting $\mathcal{N}(\cdot)$ represent the null space of a 
matrix, one has $\mathcal{N}\left(\boldsymbol{B}_{\mathcal{G}}(\boldsymbol{q})\right)=\mathcal{N}\left(\boldsymbol{B}_{\mathcal{K}_{N}}(\boldsymbol{q})\right)$ where $\mathcal{K}_{N}$ is the complete directed graph. Two frameworks $(\mathcal{G}, \boldsymbol{q})$ and $\left(\mathcal{G}, \boldsymbol{q}^{\prime}\right)$ are bearing equivalent (BE) if $\boldsymbol{\beta}_{i j}(\boldsymbol{q})=\boldsymbol{\beta}_{i j}\left(\boldsymbol{q}^{\prime}\right) \forall(i, j) \in \mathcal{E}$ and bearing congruent (BC) if $\boldsymbol{\beta}_{i j}(\boldsymbol{q})=\boldsymbol{\beta}_{i j}\left(\boldsymbol{q}^{\prime}\right) \forall i, j \in$ $\mathcal{V}, i \neq j$. If a framework $(\mathcal{G}, \boldsymbol{q})$ is IBR, then there exists a neighbor $\mathcal{U}$ of $\boldsymbol{q}$ such that any framework $\left(\mathcal{G}, \boldsymbol{q}^{\prime}\right)$, with $\boldsymbol{q}^{\prime} \in \mathcal{U}$, that is $\mathrm{BE}$ to $(\mathcal{G}, \boldsymbol{q})$ is also $\mathrm{BC}$ to $(\mathcal{G}, \boldsymbol{q})$.

It can be shown [8] that for a IBR framework in $\mathbb{R}^{3} \times \mathcal{S}^{1}$, $\operatorname{rank}\left(\boldsymbol{B}_{\mathcal{G}}(\boldsymbol{q})\right)=4 N-5$ and an orthogonal basis for the 5-dimensional null space can be explicitly expressed as [18]

$$
\begin{aligned}
\mathcal{N}\left(\boldsymbol{B}_{\mathcal{G}}^{\mathcal{W}}(\boldsymbol{q})\right) & =\operatorname{span}\left\{\left[\begin{array}{c}
\mathbf{1}_{N \otimes 3} \\
\mathbf{0}
\end{array}\right],\left[\begin{array}{c}
\boldsymbol{p}-\mathbf{1}_{N \otimes 3} \bar{p} \\
\mathbf{0}
\end{array}\right],\left[\begin{array}{c}
\left(\boldsymbol{I}_{N} \otimes \boldsymbol{S}\right)\left(\boldsymbol{p}-\mathbf{1}_{N \otimes 3} \overline{\boldsymbol{p}}\right) \\
\mathbf{1}_{N}
\end{array}\right]\right\} \\
& =\operatorname{span}\left\{\boldsymbol{n}_{1}, \boldsymbol{n}_{2}, \boldsymbol{n}_{3}\right\},
\end{aligned}
$$

where $\mathbf{1}_{N}$ is the $N$-dimensional vector of all ones, $\mathbf{1}_{N \otimes 3}=$ $\mathbf{1}_{N} \otimes \boldsymbol{I}_{3}, \overline{\boldsymbol{p}}=\mathbf{1}_{N \otimes 3}^{T} \boldsymbol{p} / N$ is the barycenter of $\boldsymbol{p}$ and $\otimes$ represents the matrix Kronecker product. The terms in (5) correspond to the three rigid-body translations $\left(\boldsymbol{n}_{1}\right)$, a dilation $\left(\boldsymbol{n}_{2}\right)$ w.r.t. $\bar{p}$, and a coordinated rotation $\left(\boldsymbol{n}_{3}\right)$ about a vertical axis passing through $\bar{p}$.

\section{B. Localization from bearing and distance measurements}

As shown in [18], a decentralized estimation algorithm can be designed for retrieving an estimation of a time-varying framework configuration $\boldsymbol{q}(t)$ using only bearing measurements and a single distance measurement. Let $\widehat{\boldsymbol{q}}=(\widehat{\boldsymbol{p}}, \widehat{\boldsymbol{\psi}})$ be an estimation of the true $\boldsymbol{q}, \boldsymbol{e}_{L}(\boldsymbol{q}, \widehat{\boldsymbol{q}})=\boldsymbol{\beta}_{\mathcal{G}}(\widehat{\boldsymbol{q}})-\boldsymbol{\beta}_{\mathcal{G}}(\boldsymbol{q})$ be the bearing localization error, and $\widetilde{d}_{\iota \kappa}=\widehat{\boldsymbol{p}}_{\iota \kappa}^{T} \widehat{\boldsymbol{p}}_{\iota \kappa}-d_{\iota \kappa}^{2}$ be the error between the estimated and true squared distances from agent $\iota$ to agent $\kappa$, which is the pair assumed able to measure its inter-distance. If $(\mathcal{G}, \boldsymbol{q})$ is IBR, the estimator

$\dot{\widehat{\boldsymbol{q}}}=k_{L} \boldsymbol{B}_{\mathcal{G}}^{\mathcal{W}}(\widehat{\boldsymbol{q}})^{T} \boldsymbol{\beta}_{\mathcal{G}}(\boldsymbol{q})+\left[\begin{array}{cc}\operatorname{diag}\left(\boldsymbol{R}_{z}\left(\widehat{\psi}_{i}\right)\right) & \mathbf{0} \\ \mathbf{0} & \boldsymbol{I}_{N}\end{array}\right]\left[\begin{array}{c}\boldsymbol{u} \\ \boldsymbol{w}\end{array}\right]-k_{d} \widetilde{d}_{\iota \kappa} \boldsymbol{D}_{\iota \kappa} \widehat{\boldsymbol{q}}$, with $\left.D_{\iota \kappa}={ }_{\kappa} \iota \begin{array}{ccccc}0 & 0 & 0 & 0 & 0 \\ 0 & -\boldsymbol{I}_{3} & 0 & \boldsymbol{I}_{3} & 0 \\ 0 & 0 & 0 & 0 & 0 \\ 0 & \boldsymbol{I}_{3} & 0 & -\boldsymbol{I}_{3} & 0 \\ 0 & 0 & 0 & \mathbf{0} & 0\end{array}\right]$,

guarantees local convergence of $\widehat{\boldsymbol{q}}$ to a configuration that is BC to $q$.

In the absence of a distance measurement $d_{\iota \kappa}$, the correct scale of the formation cannot be obviously retrieved. As discussed in [18], a wrong formation scale will, among others, also prevent convergence of (6) in case of nonstationary agents. Measuring $d_{\iota \kappa}$ allows recovering the correct formation scale, but it also requires additional sensors that are usually not available on most UAV platforms. As explained, a different possibility is to exploit, instead, an alternative metric measurement already available (without additional sensor requirements) to each agent, i.e., the bodyframe linear velocity $\boldsymbol{u}_{i}$. Section III-C will show how to exploit a SfM technique in order to estimate, from the locally available bearing and velocity measurements, a suitable set of inter robot distances to be plugged in (6).

\section{Inter-agent distance estimation from motion}

Exploiting the body-frame rigidity matrix (3-4), the dynamics of the bearing vector $\boldsymbol{\beta}_{i j}$ can be linearly expanded w.r.t. the unknown inverse depth $\chi_{i j}=1 / d_{i j}$ as follows

$$
\dot{\boldsymbol{\beta}}_{i j}=-\boldsymbol{S} \boldsymbol{\beta}_{i j} w_{i}+\boldsymbol{P}_{i j} \boldsymbol{u}_{i j} \chi_{i j}
$$

where $\boldsymbol{u}_{i j}={ }^{i} \boldsymbol{R}_{j} \boldsymbol{u}_{j}-\boldsymbol{u}_{i}$ is the relative velocity between agents $i$ and $j$ expressed in the body frame of agent $i$.

Given (7) and following [29], a (decentralized) SfM estimator for $\chi_{i j}$ can be devised as

$$
\left\{\begin{array}{l}
\dot{\hat{\boldsymbol{\beta}}}_{i j}=-\boldsymbol{S} \boldsymbol{\beta}_{i j} w_{i}+\boldsymbol{\Omega}_{i j}\left(\boldsymbol{\beta}_{i j}, \boldsymbol{u}_{i j}\right)^{T} \widehat{\chi}_{i j}-\boldsymbol{H} \boldsymbol{\xi}_{i j} \\
\dot{\hat{\chi}}_{i j}=-\widehat{\chi}_{i j}^{2} \boldsymbol{u}_{i j}^{T} \boldsymbol{\beta}_{i j}-\alpha \boldsymbol{\Omega}_{i j}\left(\boldsymbol{\beta}_{i j}, \boldsymbol{u}_{i j}\right) \boldsymbol{\xi}_{i j}
\end{array}\right.
$$

where $\boldsymbol{\Omega}_{i j}\left(\boldsymbol{\beta}_{i j}, \boldsymbol{u}_{i j}\right)=\boldsymbol{u}_{i j}^{T} \boldsymbol{P}_{i j}, \boldsymbol{\xi}_{i j}=\widehat{\boldsymbol{\beta}}_{i j}-\boldsymbol{\beta}_{i j}$ and $\boldsymbol{H}, \alpha>$ 0 are free gains. Observer (8) can be proved to be almost globally exponentially stable if and only if (iff) the following Persistency of Excitation (PE) condition holds (see e.g. [30])

$$
\int_{t}^{t+T} \boldsymbol{\Omega}_{i j}(\tau) \boldsymbol{\Omega}_{i j}^{T}(\tau) d \tau \geq \gamma>0, \quad \forall t \geq t_{0} .
$$

Note that algorithm (8) needs access to the relative agent velocity $\boldsymbol{u}_{i j}$. While both $\boldsymbol{u}_{i}$ and $\boldsymbol{u}_{j}$ are known, since the agents do not have access to a common frame, $\psi_{i j}$ is not directly available in general. An estimation $\widehat{\psi}_{i j}=\widehat{\psi}_{j}-\widehat{\psi}_{i}$ could be employed by exploiting the current state of (6) exchanged between the two neighbors over local communication. This would, however, inject the dynamics of (6) in (8), thus affecting the SfM convergence properties. On the other hand, for all edges in the undirected subgraph $\mathcal{G}_{u}$, the relative orientation $\psi_{i j}$ can be directly recovered from the available measurements $\left(\boldsymbol{\beta}_{i j}, \boldsymbol{\beta}_{j i}\right)$, exchanged over local communication, by exploiting a simple algebraic computation (see [11]), provided that $\boldsymbol{\beta}_{i j} \neq(0,0, \pm 1)$. This allows to treat $\boldsymbol{u}_{i j}$ as a known/measured quantity for all such edges.

From this point on, let us assume that $\mathcal{E}_{u} \neq \emptyset$ and $\boldsymbol{\beta}_{i j} \neq(0,0, \pm 1)$ for at least one edge in $\mathcal{E}_{u}$ (presence of a pair of agents in mutual visibility and not aligned along the $z$-axis). The SfM estimator (8) can be used to recover an estimation $\widehat{d}_{i j}$ of $d_{i j}$ for all edges in $\mathcal{E}_{u}$ satisfying this condition. The estimated $\widehat{d}_{i j}$ can, then, be used as a virtual measurement in (6) by replacing the last term with $-k_{d} \sum_{(i, j) \in \mathcal{E}_{u}} \widetilde{d}_{i j} \boldsymbol{D}_{i j} \widehat{\boldsymbol{q}}$. Note that the resulting localization algorithm remains decentralized.

Let us introduce a vector $\widehat{\chi}=\left(\widehat{\chi}_{e_{u 1}} \ldots \widehat{\chi}_{e_{u\left|\mathcal{E}_{u}\right|}}\right) \in \mathbb{R}^{\left|\mathcal{E}_{u}\right|}$ obtained by stacking the inverse distances estimated by all instances (one for each edge ${ }^{2}$ in $\mathcal{E}_{u}$ ) of (8). Let also $\lambda_{e_{u k}}=$ $\lambda_{i j}=\boldsymbol{\Omega}_{i j} \boldsymbol{\Omega}_{i j}^{T}=\boldsymbol{u}_{i j}^{T} \boldsymbol{P}_{i j} \boldsymbol{u}_{i j}$ and $\boldsymbol{\lambda}=\left(\lambda_{e_{u}} \ldots \lambda_{e_{u\left|\mathcal{E}_{u}\right|}}\right) \in$ $\mathbb{R}^{\left|\mathcal{E}_{u}\right|}$. For simplicity of notation, let us write $\lambda_{i}=\lambda_{e_{u i}}$ and assume that the edges $\mathcal{E}_{u}$ are ordered by ascending value ${ }^{3}$ of $\lambda_{i}$. Following [25], one can show that the transient response of the SfM estimation error $\widetilde{\chi}=\widehat{\chi}-\chi$ results dictated by the eigenvalues $\alpha \boldsymbol{\lambda}$.

\footnotetext{
${ }^{2}$ Having both agents $i$ and $j$ estimating the same physical quantity $d_{i j}$ introduces an unnecessary computational effort. This can be easily avoided if the two agents agree, at the startup phase, on which one should perform the estimation and communicate the result to its neighbor. A possible criterion is to, e.g., let the agent with the lower ID to perform the estimation.

${ }^{3}$ Existence of a common ordering is not required for the implementation.
} 
For a given choice of $\alpha$ (a free parameter), the larger $\lambda_{i j}$ the faster the error convergence. In particular one clearly has $\lambda_{i j} \propto\left\|\boldsymbol{u}_{i j}\right\|^{2}$ with $\lambda_{i j}=0$ if $\left\|\boldsymbol{u}_{i j}\right\|^{2}=0$ (as well-known, only a translating camera can perform SfM estimation). More in general, $\lambda_{i j}$ will also be determined by the direction of $\boldsymbol{u}_{i j}$ w.r.t. $\boldsymbol{\beta}_{i j}$. Therefore, it is meaningful to actively optimize online $\boldsymbol{u}_{i j}$, a controllable quantity, as a function of the current measurement $\boldsymbol{\beta}_{i j}$ so as to maximize the estimation performance. Sect. IV will explain how to achieve this goal in a decentralized way.

Finally, we report another interesting property of (8) that will be exploited in Sect. IV-B to adaptively tune the observability maximization. The following measurable quantity

$$
E=\sum_{(h, k) \in \mathcal{E}_{u}} E_{h k}(t)=\sum_{(h, k) \in \mathcal{E}_{u}}\left(\frac{1}{T} \int_{t-T}^{t} \boldsymbol{\xi}_{h k}^{T}(\tau) \boldsymbol{\xi}_{h k}(\tau) \mathrm{d} \tau\right)
$$

where $T>0$ is an integration window, provides a measure of the uncertainty of the estimated $\widehat{\chi}$ vs. the actual $\chi$. Indeed, the following proposition is proven in the Appendix.

Proposition 1. If the camera trajectory is exciting (i.e., $\lambda_{i j}(t)>0$ ), $E_{i j}(t) \equiv 0$ iff $\widetilde{\chi}_{i j}(t) \equiv 0$ (i.e., the estimation has converged) and $E_{i j}(t)>0$ (and thus $E>0$ ) otherwise.

\section{DECENTRALIZED ACTIVE ESTIMATION OF INTER-ROBOT DISTANCES}

As anticipated in Sect. III-C, convergence of (8) is determined by the satisfaction of the PE condition (9) and, more in general, by the eigenvalues $\lambda_{i j}=\boldsymbol{\Omega}_{i j} \boldsymbol{\Omega}_{i j}^{T}$. Since, for each edge in $\mathcal{E}_{u}, \lambda_{i j}=\lambda_{i j}\left(\boldsymbol{\beta}_{i j}, \boldsymbol{u}_{i j}\right)$, one can act on $\boldsymbol{u}_{i}$ and $\boldsymbol{u}_{j}$, depending on the current value of $\boldsymbol{\beta}_{i j}$, so as to maximize $\lambda_{i j}$ and increase the convergence rate. This section, hence, addresses the topic of generating, in a decentralized way, a suitable motion policy for realizing such optimization.

We consider, in particular, two possible scenarios. First we assume, in Sect. IV-A, that the only task assigned to the agents is their mutual localization in a shared frame and, thus, that the velocity inputs $\boldsymbol{u}_{i}$ can be specified at will so as to reach this goal in a minimum time. In Sect. IVB we consider, instead, a more common situation in which the agents are also required to reach and maintain a desired bearing formation. In this case the observability optimization can still be realized, but only in the null space of the main formation control task.

\section{A. Unconstrained active perception}

Convergence of $\widehat{\chi}$ is only attained when the slowest mode, associated to $\lambda_{1}$, has converged. To reduce the convergence time of $\widehat{\chi}$, one should then attempt to maximize $\lambda_{1}$ by acting on $\boldsymbol{u}$. The gradient of each $\lambda_{k}$ w.r.t. $\boldsymbol{u}$ can be computed in closed form if $\lambda_{k}$ has multiplicity one. For repeated eigenvalues, however, the gradient is not well defined due to reordering of the associated eigenvectors. Inspired by [24], we then propose to consider the following differentiable optimization objective:

$$
\bar{\lambda}=\|\boldsymbol{\lambda}\|_{p}=\sqrt[p]{\sum_{(h, k) \in \mathcal{E}_{u}} \lambda_{h k}^{p}}
$$

with $p \ll 0$. When $\lambda_{1} \ll \lambda_{2}$, one clearly has $\bar{\lambda} \approx \lambda_{1}$. If, instead, $\lambda_{1} \approx \lambda_{2} \approx \lambda_{m} \ll \lambda_{m+1}$, then $\bar{\lambda} \approx \sqrt[p]{m} \lambda_{1}$. In both cases, maximization of $\bar{\lambda}$ results in the maximization of the smallest eigenvalue of the system with the additional advantage of $\bar{\lambda}$ being always differentiable. Since

$$
\frac{\partial \bar{\lambda}}{\partial \boldsymbol{u}_{i}}=\sum_{[(i, j)] \in \mathcal{E}_{u}} \frac{\partial \bar{\lambda}}{\partial \lambda_{i j}} \frac{\partial \lambda_{i j}}{\partial \boldsymbol{u}_{i j}} \frac{\partial \boldsymbol{u}_{i j}}{\partial \boldsymbol{u}_{i}}=-2 \sum_{[(i, j)] \in \mathcal{E}_{u}}\left(\frac{\lambda_{i j}}{\bar{\lambda}}\right)^{p-1} \boldsymbol{u}_{i j}^{T} \boldsymbol{P}_{i j}
$$

one can use the following control law to maximize $\bar{\lambda}$ while maintaining a desired norm of $\boldsymbol{u}_{i}$ :

$$
\dot{\boldsymbol{u}}_{i}=-\frac{k_{\nu}}{2} \frac{\nu_{i}-\nu^{*}}{\nu_{i}} \boldsymbol{u}_{i}+k_{\bar{\lambda}}\left(\boldsymbol{I}_{3}-\frac{\boldsymbol{u}_{i} \boldsymbol{u}_{i}^{T}}{2 \nu_{i}}\right) \frac{\partial \bar{\lambda}^{T}}{\partial \boldsymbol{u}_{i}}
$$

where $k_{\nu}, k_{\bar{\lambda}}>0$ and $\nu_{i}=\frac{1}{2} \boldsymbol{u}_{i}^{T} \boldsymbol{u}_{i}$ is the current (squared) linear velocity norm, while $\nu^{*}$ is its desired value. Since $\bar{\lambda} \propto$ $\|\boldsymbol{u}\|^{2}$, the first term in (12) is necessary for maintaining a constant $\|\boldsymbol{u}\|$ and obtaining a fair comparison with alternative motion strategies. The second term, instead, modifies the direction of $\boldsymbol{u}_{i}$ on-line so as to maximize $\bar{\lambda}$. Note that this term disappears if $\lambda_{i j} \gg \bar{\lambda}$ because $\left(\lambda_{i j} / \bar{\lambda}\right)^{p-1} \approx 0$ in this case. On the other end, the observability maximization is fully active for the two agents involved in the reciprocal edge corresponding to $\lambda_{1}$ since, if $\lambda_{1} \ll \lambda_{2},\left(\lambda_{1} / \bar{\lambda}\right)^{p-1} \approx 1$.

We note that, of all the terms that appear in (12), the only one not directly available to agent $i$ (via direct measurement or communication with its neighbors) is $\bar{\lambda}$. A decentralized estimation of $\bar{\lambda}$ can, however, be recovered using an average consensus estimator (ACE) [31].

Finally (12) needs to be initialized with a value of $\boldsymbol{u}_{i}\left(t_{0}\right)$. One very simple initialization strategy is for each robot to randomly generate $\boldsymbol{u}_{i}\left(t_{0}\right)$ such that $\frac{1}{2} \boldsymbol{u}_{i}\left(t_{0}\right)^{T} \boldsymbol{u}_{i}\left(t_{0}\right)=\nu^{*}$.

\section{B. Bearing-constrained active perception}

Let $(\mathcal{G}, \boldsymbol{q})$ be IBR and $\left(\mathcal{G}_{d}, \boldsymbol{q}_{d}\right)$ represent a desired IBR framework. We assume that a bearing control algorithm, such as the one proposed in [26], is used for reaching a configuration BC to $\boldsymbol{q}_{d}$ by generating some suitable control inputs $\left(\boldsymbol{u}^{F}, \boldsymbol{w}^{F}\right)$. In this scenario, the optimization of $\bar{\lambda}$ can only be performed without violating the constraint $\boldsymbol{\beta}(\boldsymbol{q})=$ $\boldsymbol{\beta}\left(\boldsymbol{q}_{d}\right)$, i.e. by exploiting velocities in the null-space (5).

Motions along $\boldsymbol{n}_{1}$ result, however, in all the agents translating along parallel trajectories $\left(\dot{\boldsymbol{p}}_{i}=\boldsymbol{\nu} \forall i \in \mathcal{V}\right)$ therefore implying $\boldsymbol{u}_{i j}=\mathbf{0}_{3}, \forall(i, j) \in \mathcal{E}$ and thus $\lambda_{i}=\bar{\lambda}=0$. Similarly, $\boldsymbol{n}_{2}$ represents a scaling of the entire formation and thus it implies $\boldsymbol{u}_{i j} \| \boldsymbol{\beta}_{i j}, \forall(i, j) \in \mathcal{E}$ and, again, $\lambda_{i}=$ $\bar{\lambda}=0$. One can then conclude that, within $\mathcal{N}\left(\boldsymbol{B}_{\mathcal{G}}^{\mathcal{W}}\right)$, only $\boldsymbol{n}_{3}$ (rotation of the formation around its barycenter) results in a non-zero observability $(\bar{\lambda}>0)$. To reduce the control effort, hence, any motion along $\boldsymbol{n}_{1}$ and $\boldsymbol{n}_{2}$ should be avoided. By doing so, one also gains the additional advantage of keeping the formation scale and barycenter position constant during the active optimization, with obvious benefits, e.g., in terms of reduction of potential hazards in presence of obstacles.

In order to ensure a positive observability, while still maintaining a configuration $\mathrm{BC}$ to $\boldsymbol{q}_{d}$, and minimizing the control effort, one can, then, add to the formation control input 
$\left(\boldsymbol{u}^{F}, \boldsymbol{w}^{F}\right)$ the additional term $w_{\bar{\lambda}} \operatorname{diag}\left(\boldsymbol{R}_{1}^{T}, \ldots, \boldsymbol{R}_{N}^{T}, \boldsymbol{I}_{N}\right) \boldsymbol{n}_{3}$ where $w_{\bar{\lambda}} \in \mathbb{R}$ is the only remaining DOF for excitation maximization. The resulting control law, for agent $i$, is:

$$
\left\{\begin{array}{l}
\boldsymbol{u}_{i}=\boldsymbol{u}_{i}^{F}+w_{\bar{\lambda}} \boldsymbol{R}_{i}^{T} \boldsymbol{S}\left(\boldsymbol{p}_{i}-\overline{\boldsymbol{p}}\right) \\
w_{i}=w_{i}^{F}+w_{\bar{\lambda}}
\end{array} .\right.
$$

As for the angular velocity $w_{\bar{\lambda}}$, from (13) it follows that

$$
\frac{\partial \bar{\lambda}}{\partial w_{\bar{\lambda}}}=\sum_{i} \frac{\partial \bar{\lambda}}{\partial \boldsymbol{u}_{i}} \frac{\partial \boldsymbol{u}_{i}}{\partial w_{\bar{\lambda}}}=\sum_{i} \frac{\partial \bar{\lambda}}{\partial \boldsymbol{u}_{i}} \boldsymbol{R}_{i}^{T} \boldsymbol{S}\left(\boldsymbol{p}_{i}-\overline{\boldsymbol{p}}\right)=\sum_{i}(\delta \bar{\lambda})_{i}
$$

and an update law for $w_{\bar{\lambda}}$ can be devised as

$$
\dot{w}_{\bar{\lambda}}=-k_{w} w_{\bar{\lambda}}+k_{\bar{\lambda}} \frac{\gamma}{\gamma+\bar{\lambda}} \frac{\partial \bar{\lambda}^{T}}{\partial w_{\bar{\lambda}}}
$$

where $k_{w}, k_{\bar{\lambda}}, \gamma>0$. Note that, unlike (12), (13-15) do not enforce a constant linear velocity norm for the agents. The reason for this choice is that, in this case, since $\boldsymbol{n}_{3}$ is orthogonal to the formation control input $\left(\boldsymbol{u}^{F}, \boldsymbol{w}^{F}\right)$, it would not be possible to introduce the null-space motions and, at the same time, maintain the same $\|\boldsymbol{u}\|$ without also modifying the formation control task $\left(\boldsymbol{u}^{F}, \boldsymbol{w}^{F}\right)$. We still aim, however, at keeping a bounded $\|\boldsymbol{u}\|$. This is exactly the purpose of the damping term $-k_{w} w_{\bar{\lambda}}$ and the factor $\gamma /(\gamma+\bar{\lambda})$ in (15).

The strategy described so far would induce a persistent rotation of the formation around its barycenter so as to always guarantee a certain level of excitation for the SfM observer (8) even after the estimation error $\widetilde{\chi}(t)=\widehat{\chi}(t)-$ $\chi(t)$ has converged to zero. For some applications this might be undesirable. A more efficient strategy can be obtained by implementing (15) only when strictly needed, e.g., as long as the estimation error $\tilde{\boldsymbol{\chi}}(t)$ is larger than some minimum threshold. Obviously $\widetilde{\chi}(t)$ is not directly measurable, nevertheless, as discussed in Sect. III-C, one can leverage knowledge of the measurable quantity $E(t)$ in (10) for obtaining an insight of the current status of the estimation. Let then $0 \leq \underline{E}<\bar{E}$ be a fixed minimum/maximum threshold for $E(t)$ and define

$$
k_{E}(E):[\underline{E}, \bar{E}] \mapsto[0,1]
$$

as a monotonically increasing smooth map. Controller (15) can be modified by setting $k_{\bar{\lambda}}=k_{\bar{\lambda}}^{\prime} k_{E}(E)$ with $k_{\bar{\lambda}}^{\prime}>0$ for smoothly activating/deactivating the optimization of $\bar{\lambda}$ as a function of the current value $E$.

A possible decentralized implementation of (13-15) is represented by the block diagram in Fig. 1. Note how, because of the number of "global" quantities involved, the algorithm is significantly more involved than (12). The results of Sect. VB show, however, good performance despite the complexity.

\section{SIMULATION RESULTS}

In this section we report some simulation results for a team of $N=6$ quadrotor UAVs in the two scenarios considered in Sect. IV. We considered the full 6-DOF dynamics of quadrotor UAVs in order to test the robustness of the proposed strategy against the simplifications introduced by model (1). A low-level controller is in charge of tracking the

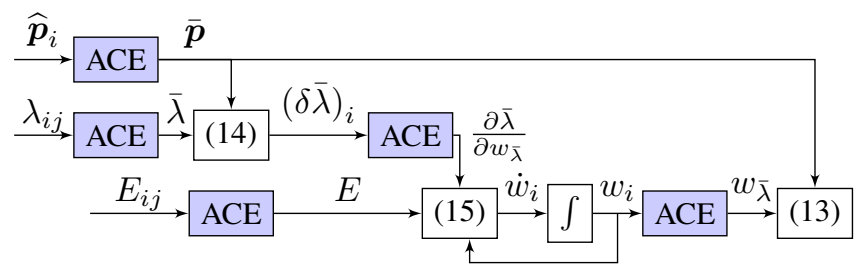

Fig. 1: Decentralized implementation of the bearing-constrained active perception strategy using average consensus estimators (ACE) [31].

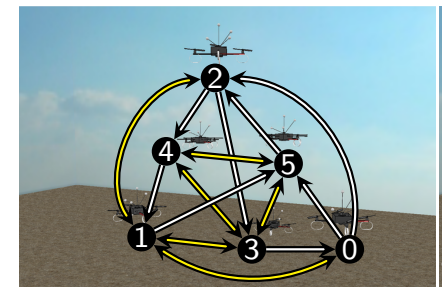

(a)

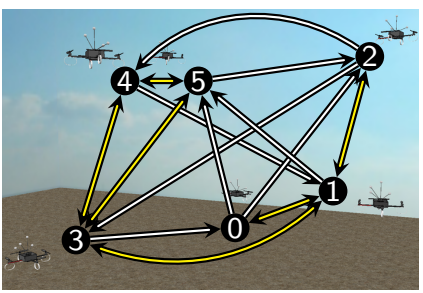

(b)
Fig. 2: (a): initial agent configuration for all simulations. (b): desired bearing formation for the simulations in Sect. V-B. An arrow from agent $i$ to $j$ indicates the availability of $\boldsymbol{\beta}_{i j}$. Reciprocal edges, for which the distance is estimated, are highlighted in yellow.

velocity reference $\boldsymbol{u}_{i}$ calculated by the control algorithms described in this paper.

The simulations were run with a fixed time step of $2 \mathrm{~ms}$ also used as sampling time for all communications between the agents. The bearing measurements $\boldsymbol{\beta}_{i j}$, on the other hand, where down-sampled at $50 \mathrm{~Hz}$ for mimicking an actual onboard camera. The following parameter set was used. $k_{L}=100$ and $k_{d}=1$ in (6). $\alpha=10$ and $\boldsymbol{H}=\boldsymbol{V} \boldsymbol{C} \boldsymbol{V}^{T}$ with $\boldsymbol{C}=\operatorname{diag}\left(2 \sqrt{\alpha \lambda_{i j}}, 10,10\right)$ where $\boldsymbol{\Omega}_{i j}=\boldsymbol{U} \boldsymbol{S} \boldsymbol{V}^{T}$ is the current SVD decomposition of $\boldsymbol{\Omega}_{i j}$ in (8) (see [25]). $p=-5$ in (11). $k_{\nu}=10, \nu^{*}=0.25, k_{\bar{\lambda}}=15$ in (12). $k_{w}=1, \gamma=0.05$ and $k_{\bar{\lambda}}^{\prime}=2$ in (15). $T=1 \mathrm{~s}$ in (10) and $\underline{E}=1 \times 10^{-4}$ and $\bar{E}=1 \times 10^{-3}$ in (16).

The initial agent configuration, represented in Fig. 2a, was the same for all simulation cases. The initial state $\widehat{\boldsymbol{q}}\left(t_{0}\right)$ of the localization observer (6) was also common to all simulations and was used to initialize the SfM observer state $\widehat{\chi}\left(t_{0}\right)$. The desired bearing configuration for (13), used for the simulations in Sect. V-B, is finally reported in Fig. 2b. We also invite the reader to watch the accompanying video.

\section{A. Unconstrained active perception}

The first set of simulations is meant to validate the effectiveness of the unconstrained observability maximization strategy described in Sect. IV-A. In this scenario, the robots are not required to reach and maintain any specific bearing formation. Their only goal is, instead, to obtain an accurate estimation of the formation scale during the localization task.

In Fig. 3 we report the results obtained using the active control strategy (12) (case 1 - blue lines) and those obtained by letting the robots follow random trajectories (case 2 - red lines). To obtain a fair comparison, since, as explained, the observability is proportional to the norm of $\boldsymbol{u}$, in both cases we maintained a constant and equal linear velocity norm for all the agents. This was obtained, in case 1, thanks to 


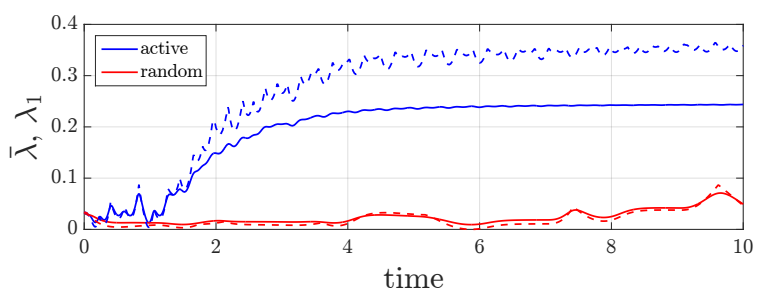

(a)

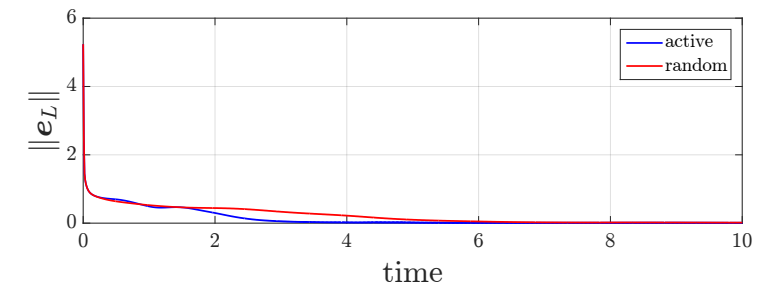

(c)

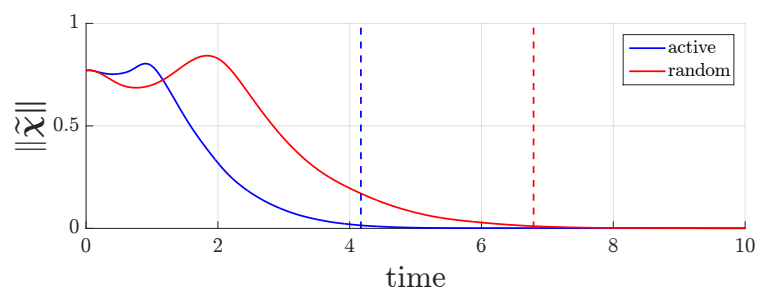

(b)

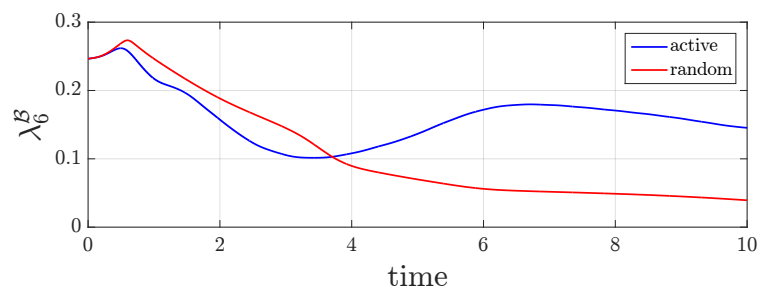

(d)

Fig. 3: Scenario I: unconstrained collaborative localization for a random motion (case 2 - red lines) and using an observability maximization strategy to maximize on-line the value of $\bar{\lambda}$ (case 1 - blue lines). (a): observability index (solid lines) and smallest eigenvalue $\lambda_{1}$ (dashed lines). Note how both are correctly increased under the effect of the observability maximization. (b): SfM estimation error $\widetilde{\chi}=\widehat{\chi}-\chi$. Vertical dashed lines represent the instants at which all components of $\tilde{\chi}$ fall below $0.01 \mathrm{~m}^{-1}$. Note how the estimation converges much faster in case 1 due to the higher value of $\bar{\lambda}$. (c): localization error. (d): smallest non-zero eigenvalue of matrix $\boldsymbol{B}_{\mathcal{G}}^{T} \mathcal{B}_{\mathcal{G}}$.

the presence of the first term in (12) and, in case 2 by an appropriate re-scaling of the randomly generated inputs $\boldsymbol{u}_{i}$.

First of all one can notice, from Fig. 3a, that the active observability maximization (the second term in (12)) resulted, as expected, in a significant increase (approximately by a factor of 10 in average) of the observability index $\bar{\lambda}$ (represented by solid lines) in case 1 w.r.t. case 2 . The plot also shows that, as predicted by the analysis, $\bar{\lambda}$ represents a reasonable approximation of the smallest eigenvalue $\lambda_{1}$ (represented by the dashed lines). Optimization of $\bar{\lambda}$ does, in fact, result in a maximization of $\lambda_{1}$ with the additional advantage of $\bar{\lambda}$ being always differentiable. Note, in fact, the presence of numerous jumps/irregularities in the evolution of $\lambda_{1}$, especially in the active case between $t=2 \mathrm{~s}$ and $t=6 \mathrm{~s}$. These jumps are due to multiple reordering of the eigenvalues because of the proximity of $\lambda_{1}$ and $\lambda_{2}$ (which often switch order during motion) and are significantly smoothened in $\bar{\lambda}$.

The higher excitation level predictably results in an increased convergence rate for the SfM observer (8). As it can be noticed from Fig. 3b, in fact, all of the estimated quantities $\chi_{i j}$ converge to an error smaller than $0.01 \mathrm{~m}^{-1}$ after only about $4.162 \mathrm{~s}$ in case 1 (vertical blue line). The same result is achieved, in case 2 , only for $t=6.788 \mathrm{~s}$ (vertical red line).

Finally, in Figs. 3c and 3d, we report the behavior of the bearing localization error $\boldsymbol{e}_{L}$ and of the smallest non-zero eigenvalue $\lambda_{6}^{\mathcal{B}}$ of matrix $\mathcal{B}_{\mathcal{G}}^{T} \mathcal{B}_{\mathcal{G}}$ for cases 1 and 2. Since, in both cases, the formation is IBR (i.e. $\lambda_{6}^{\mathcal{B}}>0$ ) and the estimated distances have converged to the actual ones, one can also conclude that $\widehat{\boldsymbol{q}}$ has converged to a configuration that not only is BC to $\boldsymbol{q}$ but it is also correctly scaled and, hence, can only differ from $\boldsymbol{q}$ by a global translation and rotation, i.e., the directions spanned by $\boldsymbol{n}_{1}$ and $\boldsymbol{n}_{3}$ in (5).

\section{B. Bearing-constrained active perception}

The second set of simulations, reported in this section, concerns the scenario investigated in Sect. IV-B. In this case we consider a more common situation w.r.t. the one analyzed in Sects. IV-A and V-A. We assume, in fact, that a primary bearing formation regulation task is assigned and the observability must be optimized (maximizing $\bar{\lambda}$ ) while also steering the formation towards a configuration that is BC to the desired one $\boldsymbol{q}_{d}$. To reduce the control effort, we also wish to limit, as much as possible, the action of the observability maximization. We do this by exploiting the quantity $E$ in (10), as explained in Sect. IV-B, to deactivate the maximization once the SfM error $\widetilde{\chi}$ has converged.

The plots in Fig. 4 report the results obtained when either implementing only the formation control law in [18] (case 3 - red lines) or when also introducing an additional observability maximization term as in (13) (case 4 - blue lines). In both cases, observer (8) is used to estimate the distances between the agents in $\mathcal{G}_{u}$. Similarly to Fig. 3a, one can notice, from Fig. 4a, that the active strategy results, also in this case, in a much larger value of the observability index $\bar{\lambda}$ in case 4 w.r.t. case 3 . More precisely, in the interval $t=[0.08,5.06] \mathrm{s}$, during which the optimization is mostly active being $k_{E}>0.5, \bar{\lambda}$ is, in average, about 25 times larger in case 4 w.r.t. case 3 . This, in turn, results in a very fast convergence of the SfM estimation error $\widetilde{\chi}$ whose components fall below the threshold of $0.01 \mathrm{~m}^{-1}$ after only $1.644 \mathrm{~s}$ in case 4 . In case 3 , instead, the observability index $\bar{\lambda}$ does not maintain a sufficiently large value before the agents stop moving due to convergence of $\boldsymbol{e}_{F}$. As a result, the estimation error $\widetilde{\chi}$ never falls below the desired threshold and one of its components settles to a value of $0.0328 \mathrm{~m}^{-1}$.

From Fig. $4 \mathrm{c}$ one can also appreciate how the active observability maximization is correctly deactivated after convergence of the estimation error $\widetilde{\chi}$. Indeed, the decrease of the measurable quantity $E$ causes the adaptive gain $k_{E} \approx 0$ to cancel the optimization term in (15). Shortly after, the common rotation command $w_{\bar{\lambda}}$ is also disabled under the effect of the damping term in (15). 


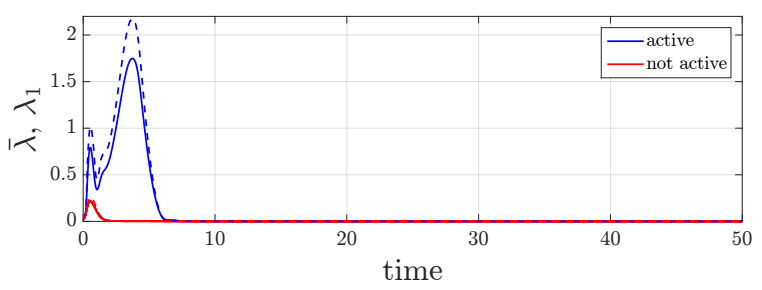

(a)

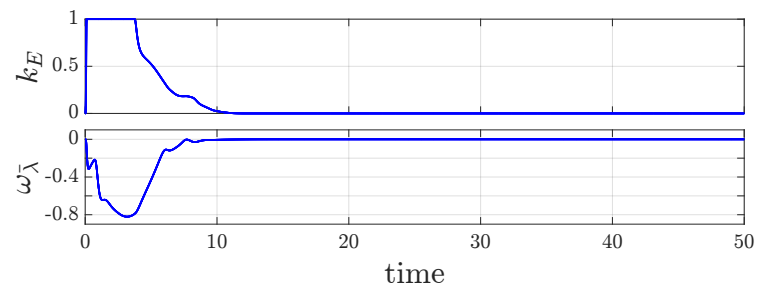

(c)

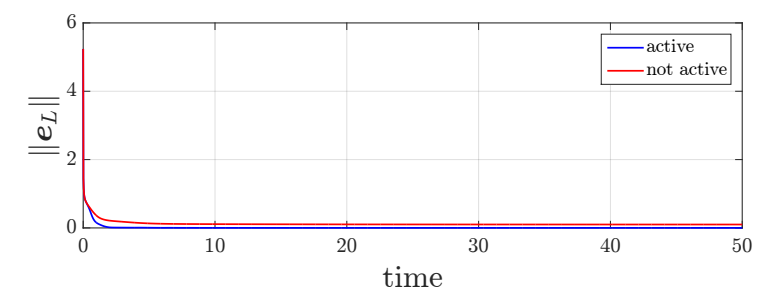

(e)

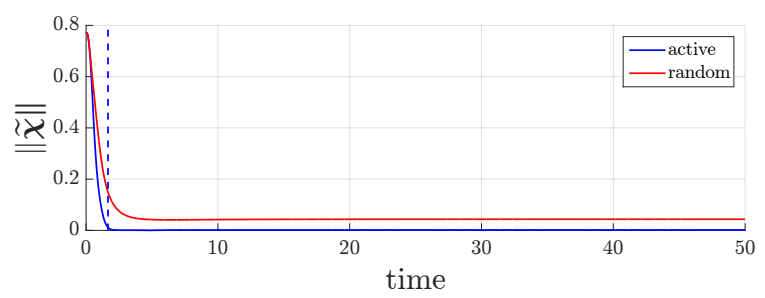

(b)

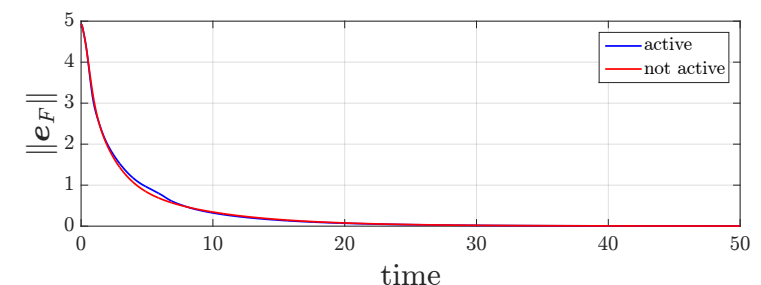

(d)

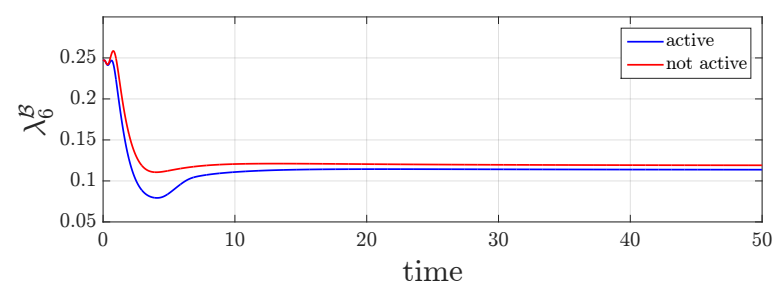

(f)

Fig. 4: Scenario II: formation control with (case 4 - blue lines) and without (case 3 - red lines) concurrent observability maximization in the null space. (a): observability index (solid lines) and smallest eigenvalue $\lambda_{1}$ (dashed lines). Note how both are correctly increased under the effect of the observability maximization. (b): SfM estimation error $\widetilde{\chi}=\widehat{\chi}-\chi$. A vertical dashed line represents the instant at which all components of $\widetilde{\chi}$ fall below $0.01 \mathrm{~m}^{-1}$ for case 4 . Note that such a condition is never reached in case 3 . (c): adaptive gain used for tuning the observability maximization (top) and common rotational command in the null space of $\boldsymbol{B}_{\mathcal{G}}^{\mathcal{W}}$ (bottom). (d): formation control error, note how the observability maximization correctly acts in the null space of the bearing formation controller and thus it does not significantly affect the evolution of the bearing error. (e): localization error. (f): smallest non-zero eigenvalue of matrix $\mathcal{B}_{\mathcal{G}}^{T} \mathcal{B}_{\mathcal{G}}$.

The behavior of the localization error $\boldsymbol{e}_{L}$ and of the smallest non-zero eigenvalue $\lambda_{6}^{\mathcal{B}}$ of matrix $\boldsymbol{B}_{\mathcal{G}}^{T} \mathcal{B}_{\mathcal{G}}$ are reported, for both cases, in Figs. 4e and 4f. Both in case 3 and in case 4, the formation is IBR (i.e. $\lambda_{6}^{\mathcal{B}}>0$ ) all along the motion. Nevertheless, only in case 4 the localization error $\boldsymbol{e}_{L}$ correctly converges to zero. In fact, the incomplete convergence of the SfM observer (8), due to an insufficient excitation level of the agent trajectory, introduces a disturbance in the localization filter (6) which relies on an inconsistent set of virtual distance measurements. On the one hand, this underlines, once more, the importance of actively ensuring a sufficient observability. On the other hand, this also shows a limitation of our current approach. Running each SfM instance independently, in fact, does not ensure consistency of the estimated distances with the bearing measurements.

One naive workaround to this issue would be to always allow only a single pair of agents in $\mathcal{G}_{u}$ to estimate their relative distance. This solution, however, would not exploit all the available sensor information. Another possibility would be to exploit the measurable quantity $E$ to define the gain $k_{d}$ in (6) so as to ignore all virtual distance measurements that have not converged to a desired minimum accuracy. This would, however, complicate the design introducing additional thresholds and gains. Finally, as shown e.g. in [10], it is also possible to exploit the bearing measurements to express all inter-agent distances in terms of a single scale factor. A better solution would, then, be to have all agents estimate directly this single metric quantity. How to do this in a decentralized way and without inducing complex couplings between the dynamics of (6) and (8) is, however, still an open problem.

Before concluding this discussion, we want to stress the fact that none of these techniques can, anyway, guarantee convergence of the formation scale if the agent trajectories are not persistently exciting and, hence, they can only ensure localization convergence for stationary agents (see [18]). An active perception strategy, such as the one described in this work, would remain, therefore, equally useful.

Finally Fig. 4d shows the effectiveness of the rather complex combination of consensus filters (see Fig. 1) in ensuring a coordinated motion of the agents in the null space of the bearing constraints. The observability maximizaiton has, in fact, negligible effects on the bearing error $\boldsymbol{e}_{F}$.

\section{CONCLUSIONS}

In this paper we have presented a fully decentralized active perception strategy that allows estimating the correctly scaled configuration of a formation of moving UAVs using only body-frame bearing measurements, acquired by onboard cameras, and the agent body-frame linear velocities.

This result was obtained by coupling the bearing rigiditybased localization algorithm proposed in [8], [18] with an extension of recently developed active SfM techniques [25] to the case of multiple robots. The resulting algorithm results in a decentralized maximization of SfM performance when only 
considering the localization task and when also maintaining a desired bearing formation. The reported simulation results showed, in both cases, a significative improvement of the SfM estimation performance when employing our strategy.

Since the performance of the overall localization is also determined by the rigidity of the formation, we are currently investigating the possibility of coupling our strategy with an extension of recent rigidity maintenance control techniques [15] to the case of bearing measurements. Another interesting point, already discussed in Sect. V-B, would be to reformulate the SfM problem in terms of a single metric scale to be estimated by all agents. This would always ensure consistency of the estimated configuration with the available bearing measurements.

\section{APPENDIX}

Proof of Prop. 1. As shown in [29], observer (8) results in the following (approximate) error dynamics

$$
\left\{\begin{array}{l}
\dot{\boldsymbol{\xi}}_{i j}=-\boldsymbol{H} \boldsymbol{\xi}_{i j}+\boldsymbol{\Omega}_{i j}\left(\boldsymbol{\beta}_{i j}, \boldsymbol{u}_{i j}\right)^{T} \widetilde{\chi}_{i j} \\
\dot{\widetilde{\chi}}_{i j}=-\alpha \boldsymbol{\Omega}_{i j}\left(\boldsymbol{\beta}_{i j}, \boldsymbol{u}_{i j}\right) \boldsymbol{\xi}_{i j}
\end{array} .\right.
$$

The hypothesis $\lambda_{i j}>0$ implies full row-rankness of $\boldsymbol{\Omega}_{i j}$ and, consequently, the following holds

- if $\left\|\boldsymbol{\xi}_{i j}(t)\right\| \equiv 0$ then $\boldsymbol{\xi}(t) \equiv \mathbf{0}$ and $\dot{\boldsymbol{\xi}}_{i j}(t) \equiv \mathbf{0}$. The first row of (17) then reduces to $\boldsymbol{\Omega}_{i j}^{T} \widetilde{\chi}_{i j} \equiv \mathbf{0}$. Since $\boldsymbol{\Omega}_{i j}$ is full row-rank, this implies $\left|\widetilde{\chi}_{i j}(t)\right| \equiv 0$;

- if $\left|\widetilde{\chi}_{i j}(t)\right| \equiv 0$, the first row of (17) reduces to $\dot{\boldsymbol{\xi}}_{i j}=$ $-\boldsymbol{H} \boldsymbol{\xi}_{i j}$. Being $\boldsymbol{H}$ positive definite, it follows that, at steady-state, the only possible solution is $\boldsymbol{\xi}_{i j}(t) \equiv \mathbf{0}$.

These two implications then prove that $\left\|\boldsymbol{\xi}_{i j}(t)\right\| \equiv 0 \Longleftrightarrow$ $\left|\widetilde{\chi}_{i j}(t)\right| \equiv 0$. By logical negation one can also prove that $\left|\tilde{\chi}_{i j}(t)\right|>0$ a.e. $\Longrightarrow\left\|\boldsymbol{\xi}_{i j}(t)\right\|>0$ a.e. and $\left\|\boldsymbol{\xi}_{i j}(t)\right\|>0$ a.e. $\Longrightarrow\left|\tilde{\chi}_{i j}(t)\right|>0$ a.e.. The proof is concluded by considering that $E_{i j}(t)$ is the moving average of $\left\|\boldsymbol{\xi}_{i j}(t)\right\|^{2}$.

\section{REFERENCES}

[1] D. Fox, J. Ko, K. Konolige, B. Limketkai, D. Schulz, and B. Stewart, "Distributed Multirobot Exploration and Mapping," Proc. of the IEEE, vol. 94, no. 7, pp. 1325-1339, 2006.

[2] J. S. Jennings, G. Whelan, and W. F. Evans, "Cooperative Search and Rescue with a Team of Mobile Robots," in 8th Int. Conf. on Advanced Robotics, Monterey, CA, Jul. 1997, pp. 193-200.

[3] N. Michael, J. Fink, and V. Kumar, "Cooperative Manipulation and Transportation with Aerial Robots," Autonomous Robots, vol. 30, no. 1, pp. 73-86, 2011.

[4] P. Robuffo Giordano, A. Franchi, C. Secchi, and H. H. Bülthoff, "A Passivity-Based Decentralized Strategy for Generalized Connectivity Maintenance," Int. J. of Robotics Research, vol. 3, no. 32, pp. 299-323, 2013.

[5] A. Kushleyev, D. Mellinger, C. Powers, and V. Kumar, "Towards A Swarm of Agile Micro Quadrotors," Autonomous Robots, vol. 35, no. 4, pp. 287-300, 2013.

[6] S. Zhao and D. Zelazo, "Bearing Rigidity and Almost Global BearingOnly Formation Stabilization," IEEE Trans. on Automatic Control, vol. 61, no. 5, pp. 1255-1268, 2016.

[7] A. N. Bishop, I. Shames, and B. D. O. Anderson, "Stabilization of rigid formations with direction-only constraints," in 50th IEEE Conf. on Decision and Control, Orlando, FL, Dec. 2011, pp. 746-752.

[8] A. Franchi, C. Masone, V. Grabe, M. Ryll, H. H. Bülthoff, and P. Robuffo Giordano, "Modeling and Control of UAV Bearing Formations with Bilateral High-level Steering," Int. J. of Robotics Research, vol. 31, no. 12, pp. 1504-1525, 2012.
[9] E. Montijano, D. Zhou, M. Schwager, and C. Sagues, "Distributed formation control without a global reference frame," in American Control Conf., Portland, OR, Jun. 2014, pp. 3862-3867.

[10] D. Zelazo, A. Franchi, and P. Robuffo Giordano, "Rigidity Theory in $S E(2)$ for Unscaled Relative Position Estimation using only Bearing Measurements," in 2014 European Control Conf., 2014, pp. 27032708.

[11] A. Cornejo, A. J. Lynch, E. Fudge, S. Bilstein, M. Khabbazian, and J. McLurkin, "Scale-Free Coordinates for Multi-Robot Systems with Bearing-Only Sensors," Int. J. of Robotics Research, vol. 32, no. 12, pp. 1459-1474, 2013.

[12] B. D. O. Anderson, C. Yu, B. Fidan, and J. M. Hendrickx, "Rigid graph control architectures for autonomous formations," IEEE Control Systems Mag., vol. 28, no. 6, pp. 48-63, 2008.

[13] L. Krick, M. E. Broucke, and B. A. Francis, "Stabilisation of infinitesimally rigid formations of multi-robot networks," Int. J. of Control, vol. 82, no. 3, pp. 423-439, 2009.

[14] H. Garcia de Marina, M. Cao, and B. Jayawardhana, "Controlling Rigid Formations of Mobile Agents Under Inconsistent Measurements," IEEE Trans. on Robotics, vol. 31, no. 1, pp. 31-39, 2015.

[15] D. Zelazo, A. Franchi, H.-H. Bülthoff, and P. Robuffo Giordano, "Decentralized rigidity maintenance control with range measurements for multi-robot systems," Int. J. of Robotics Research, vol. 34, no. 1, pp. 105-128, 2015.

[16] I. Shames, A. N. Bishop, and B. D. O. Anderson, "Analysis of Noisy Bearing-Only Network Localization," IEEE Trans. on Automatic Control, vol. 58, no. 1, pp. 247-252, 2013.

[17] R. Tron, L. Carlone, F. Dellaert, and K. Daniilidis, "Rigid components identification and rigidity enforcement in bearing-only localization using the graph cycle basis," in American Control Conf., Chicago, IL, Jul. 2015, pp. 3911-3918.

[18] F. Schiano, A. Franchi, D. Zelazo, and P. Robuffo Giordano, "A Rigidity-Based Decentralized Bearing Formation Controller for Groups of Quadrotor UAVs," in 2016 IEEE/RSJ Int. Conf. on Intelligent Robots and Systems, Daejeon, Korea, Oct. 2016.

[19] X. S. Zhou and S. I. Roumeliotis, "Determining 3-D Relative Transformations for Any Combination of Range and Bearing Measurements," IEEE Trans. on Robotics, vol. 29, no. 2, pp. 458-474, 2013.

[20] Y. Ma, S. Soatto, J. Kosecka, and S. Sastry, An invitation to $3 D$ vision. Springer, 2003.

[21] A. Martinelli, F. Pont, and R. Siegwart, "Multi-robot localization using relative observations," in 2005 IEEE Int. Conf. on Robotics and Automation, Barcelona, Spain, Apr. 2005, pp. 2797-2802.

[22] A. Cristofaro and A. Martinelli, "Optimal Trajectories for Multi Robot Localization," in 49th IEEE Conf. on Decision and Control, Atlanta, GA, Dec. 2010, pp. 6358-6364.

[23] M. W. Achtelik, S. Weiss, M. Chli, and R. Siegwart, "Path Planning for Motion Dependent State Estimation on Micro Aerial Vehicles," in 2013 IEEE Int. Conf. on Robotics and Automation, Karlsruhe, Germany, May 2013, pp. 3926-3932.

[24] F. Morbidi and G. L. Mariottini, "Active Target Tracking and Cooperative Localization for Teams of Aerial Vehicles," IEEE Trans. on Control Systems Technology, vol. 21, no. 5, pp. 1694-1707, 2013.

[25] R. Spica and P. Robuffo Giordano, "A Framework for Active Estimation: Application to Structure from Motion," in 52nd IEEE Conf. on Decision and Control, Florence, Italy, Dec. 2013, pp. 7647-7653.

[26] D. Zelazo, P. Robuffo Giordano, and A. Franchi, "Bearing-Only Formation Control Using an SE(2) Rigidity Theory," in 54th IEEE Conf. on Decision and Control, Osaka, Japan, Dec. 2015, pp. 61216126.

[27] C. D. Godsil and G. Royle, Algebraic Graph Theory. Springer, 2001.

[28] A. Franchi, G. Oriolo, and P. Stegagno, "Mutual Localization in MultiRobot Systems using. Anonymous Relative Measurements," Int. J. of Robotics Research, vol. 32, no. 11, pp. 1302-1322, 2013.

[29] A. De Luca, G. Oriolo, and P. Robuffo Giordano, "Feature depth observation for image-based visual servoing: Theory and experiments," Int. J. of Robotics Research, vol. 27, no. 10, pp. 1093-1116, 2008.

[30] R. Marino and P. Tomei, Nonlinear Control Design: Geometric, Adaptive and Robust. Prentice Hall, 1995.

[31] R. Freeman, P. Yang, and K. Lynch, "Stability and convergence properties of dynamic average consensus estimators," in 45th IEEE Conf. on Decision and Control, 2006, pp. 338-343. 